\title{
How to Achieve Approval of Radiolabeled-PSMA Diagnostic and Therapeutic Agents
}

\author{
Michael M. Graham \\ Department of Radiology, University of Iowa Hospitals and Clinics, Iowa City, Iowa
}

$\mathbf{T}$

he theranostic combination of ${ }^{68} \mathrm{Ga}$-labeled diagnostic agents with ${ }^{90} \mathrm{Y}$ - or ${ }^{177} \mathrm{Lu}$-labeled therapeutic somatostatin ligands has been shown to be a remarkably accurate and effective way to characterize and treat neuroendocrine tumors. Food and Drug Administration approval of such agents is expected to occur within the next year.

The success of a similar combination of ${ }^{68} \mathrm{Ga}$ and ${ }^{177} \mathrm{Lu}$ agents has now been demonstrated for diagnosis and therapy of prostate cancer with small-molecule prostate-specific membrane antigen (PSMA)-targeted ligands. The initial results have been extraordinarily promising, but development and approval of these agents is being slowed significantly by the expense of funding the necessary clinical trials and by fragmentation of current efforts due to several competing compounds. Furthermore, the most commonly studied ${ }^{68} \mathrm{Ga}$-labeled compound appears to be unpatented and so is not supported by any large company that might have the resources to conduct the trials needed to bring the agent to approval.

This situation is frustrating for our patients, who are well aware of these highly promising agents and are eagerly awaiting their wider availability, and for the entire nuclear medicine community, who would like to assess and treat these patients more effectively. A recent editorial by authors from The Netherlands and Germany "call[s] on the nuclear medicine community not to waste this unique opportunity to greatly enlarge the role of nuclear medicine by the practice of the same petty rivalry that in the past could be seen so often" (1).

In the past few years most, if not all, large radiopharmaceutical companies have withdrawn from the market because the large financial investment cannot be justified by the limited expected return. What remains are several more tightly focused medium and small companies that are trying to bring new agents to approval. However, the cost of trials and the onerous path to approval have slowed or stopped their efforts. An exception is the case of a definite diagnostic-therapeutic pair, ${ }^{68} \mathrm{Ga}$ - and ${ }^{177} \mathrm{Lu}$-DOTATATE, which is likely to be approved relatively soon.

Received Mar. 18, 2016; revision accepted Mar. 22, 2016.

For correspondence or reprints contact: Michael M. Graham, Division of Nuclear Medicine, Department of Radiology, Room 3863 JPP, University of lowa Hospitals and Clinics, 200 Hawkins Dr., lowa City, IA 52242.

E-mail: michael-graham@uiowa.edu

Published online Apr. 28, 2016.

COPYRIGHT (C) 2016 by the Society of Nuclear Medicine and Molecular Imaging, Inc.

DOI: 10.2967/jnumed.116.175950
Three types of industry are interested in seeing approval of more radiopharmaceuticals such as the PSMA-directed ligands. The first is PET/CT and PET/MR imaging manufacturers, since there is a real possibility that demand for new scanners will increase; the second is large radiopharmacy companies, since collaboration with academia would likely both accelerate the approval process and save money; and the third is smaller radiopharmacy companies, which may be unable to raise the resources to fulfill all the requirements for a new-drug application without help.

Several approaches to addressing this problem are being explored. One is simply to find more money. It is possible that a broadly organized collaboration between academia and industry could successfully persuade one of the large health caredirected or patient advocacy foundations to help fund a joint effort to bring a theranostic pair to approval. The motivations for industry are that the effort of designing and conducting the trials would be distributed and eased by the considerable assistance from academia and that the cost and risk would be reduced. The obvious motivation for academia is that the agents would become available years sooner if a collaborative approach is adopted than if the current fragmented approach continues.

Another approach is for academia to address the problem without industry support. An example that is already under way is the Gallium Users Group, which is part of the Clinical Trials Network of the Society of Nuclear Medicine and Molecular Imaging. This group comprises several academic sites that together are beginning coordinated clinical trials with ${ }^{68} \mathrm{Ga}-\mathrm{PSMA}$ HBED-CC (Glu-NH-CO-NH-Lys-(Ahx)- ${ }^{68} \mathrm{Ga}-\mathrm{Ga}$ ( $N, N^{\prime}$-bis[2-hydroxy-5-(carboxyethyl)benzyl] ethylenediamine- $N$, $N^{\prime}$-diacetic acid)), an unpatented agent. Trials with a therapeutic agent will follow as soon as feasible. The goal is to design a multicenter clinical trial in which all sites have identical inclusion and exclusion criteria for at least a fraction of the subjects, along with a standardized imaging protocol and radiopharmaceutical preparation, so that the data from all sites are harmonized and can be combined in support of a new-drug application. The studies are initially being funded locally or through cost-recovery investigational new drugs. Funding will also be sought from one or more philanthropic organizations, as well as from industry. Eventually it is likely that a commercial partner capable of packaging and distributing the agent will be found. Even though the agent is unpatented, the relatively low investment should justify the modest cost of organizing the data and submitting the new-drug application to the Food and Drug Administration, especially since the 
academic partners would be willing and eager to help bring the agent to market.

Essential to both possible approaches is a detailed discussion with the Food and Drug Administration to explicitly define what is needed to achieve approval of these agents. For the first approach, we need to organize a preliminary discussion between interested companies and representatives from academia to explore the feasibility of collaborating. For the second approach, we need to gather representatives from several academic sites to jointly plan and agree on a path forward.

Regardless of the approach chosen, it is important to move forward rapidly, since PSMA-directed ligands are clearly going to revolutionize the approach to diagnosis and treatment of metastatic prostate cancer and help thousands of patients.

\section{DISCLOSURE}

No potential conflict of interest relevant to this article was reported.

\section{REFERENCE}

1. Mottaghy FM, Behrendt FF, Verburg FA. ${ }^{68}$ Ga-PSMA-HBED-CC PET/CT: where molecular imaging has an edge over morphological imaging. Eur J Nucl Med Mol Imaging. 2016;43:394-396. 